\title{
La evaluación en las universidades andaluzas
}

\author{
Antonio Trinidad Requena \\ Universidad de Granada. Departamento de Ciencias Políticas y Sociología. \\ Granada. Spain
}

\section{Resumen}

El presente artículo aborda la evaluación de la Facultad de Ciencias Políticas y Sociología de la Universidad de Granada. Tiene como objeto clarificar el concepto de evaluación, los objetivos a cubrir y su contextualización. Igualmente, analiza los criterios en los que se basan las decisiones metodológicas adoptadas, señalando sus ventajas e inconvenientes. Por último, presentamos el modelo de evaluación empleado en el análisis de la Facultad y los resultados derivados de su aplicación.

Palabras clave: evaluación, universidad, organización, centros educativos, profesores.

Abstract. The evaluation in Andalucid's universities

This article deals with the evaluation of the Faculty of Political Science and Sociology at the University of Granada. Its object is to clarify the concept of evaluation, the objetives to be coverd and their placing into context. It also analyzes the criteria on which the selected methodological decisions are based, pointing out the advantages and drawbacks. Finally, we present the evaluation model used in the analysis of the Faculty and the results deriving from its application.

Key words: evaluation, university, organization, teaching institution, teacher.

\begin{aligned} & \multicolumn{2}{c}{ Sumario } \\ & Introducción $\begin{array}{l}\text { Descripción del modelo de evaluación } \\ \text { Cuestiones metodológicas }\end{array} \\ &$ Bibliografía \end{aligned}

\section{Introducción}

La evaluación de programas, políticas públicas, constituye un tema de máxima actualidad, como lo demuestra la cantidad de publicaciones aparecidas recientemente en el mercado editorial, tanto de libros como de artículos y sobre todo la prolífera cantidad de instrumentos de evaluación.

$Y$ es que no debemos olvidar la existencia de numerosos factores que favorecen la práctica de la evaluación. Podríamos mencionar: 
a) La integración europea. España ha afrontado en los diez últimos años, y tras una compleja transición democrática, la entrada en las instituciones europeas. La Comunidad Europea está llevando a cabo un conjunto de actividades, integradas en el Programa Monitor, tendientes a evaluar estrategias, programas, proyectos, así como el apoyo y el desarrollo de metodologías evaluativas. Todo ello ha llevado a que los responsables políticos de nuestro país presten atención a la evaluación de políticas públicas.

b) La diversidad de la intervención puiblica. Para Roses (1984), el Estado de bienestar ha desplazado el centro de la gravedad desde la «alta política» característica del viejo Estado liberal -relaciones internacionales, guerra y paz, ley y orden, finanzas públicas - a la «baja política» - consistente en ofrecer bienes y servicios a los ciudadanos donde quiera que se encuentren-. Dicho de otra forma, las administraciones públicas cubren un espectro de una extrema variedad: infraestructuras, sanidad, vivienda, educación, sistema de pensiones, subsidios, subvenciones, en la intervención en la «baja política», y sobre todo en aque:llas acciones donde los resultados no son visibles y los ciudadanos son los «clientes» de las prestaciones públicas, requiere los servicios de la evaluación como instrumento que garantice la equidad y la transparencia de la acción en áreas como la cultura, el bienestar, los equilibrios interterritoriales, etc.

c) La legitimación. La evaluación ha recibido el gran impulso como consecuencia de sus efectos también legitimadores. Y legitimadores tanto hacia dentro como hacia fuera. Es decir, como medio para obtener recursos, inversiones, etc., por ejemplo del Ministerio de Economía, y como medio, hacia fuera, para que la evaluación de los efectos de las políticas legitime que los ciudadanos contribuyen — vía impuestos- con sus recursos al sostenimiento de esas políticas.

d) El crecimiento de costes. Cuando el gasto público se dispara, los recursos disponibles son escasos y la presión fiscal es elevada. Entonces la actividad pública no puede llevarse a cabo a cualquier precio, sino con un coste adecuado. Y ello no por las razones de competitividad, sino porque el empleo de los recursos públicos - medios escasos para usos alternativosestá sujeto a una racionalidad de asignación no sólo económica, sino también política, según la cual debe maximizarse su eficacia y eficiencia. Como dice el profesor Beltrán (1991): «indagar la eficacia y la eficiencia de la acción pública puede permitir que se revisen los cursos de actividades en los que no se logren en una proporción razonable los objetivos propuestos, o en los que se utilice de forma inadecuada un volumen excesivo de los recursos disponibles, en último extremo, dinero público». En el caso que nos ocupa -instituciones universitarias - , los altos costes totales de la educación superior demandan, tanto por parte de las autoridades educativas como de la sociedad en general, un mayor control sobre las universidades.

e) La calidad. Es un concepto que en los últimos años ha impregnado a toda la sociedad. Parecería que la evaluación, cual producto de mercado, ha 
incorporado estos elementos de la coyuntura sociocultural en que vivimos, tomando la "calidad de la enseñanza" un auge hasta ahora desconocido, ensalzándose todas las modalidades del término: enseñanza de calidad, profesorado de calidad y evaluar para aumentar la calidad. Calidad como requisito para la competitividad.

Como consecuencia del interés por la calidad se está creando una predisposición hacia la evaluación que implica, no sólo una mayor y mejor evaluación de las instituciones, sino también una perspectiva que suponga, además de una verificación del funcionamiento del sistema, un método para mejorarlo.

Por lo tanto, la cuestión de la evaluación, que en estos momentos está cobrando un gran protagonismo en todos los ámbitos de intervención social, tiene una importancia especial en el de la educación, sobre todo a nivel institucional. Prueba de ello ha sido la creación en los últimos años de:

- El Instituto de Evaluación del Sistema Educativo, MEC.

- Centros y gabinetes de evaluación en diferentes universidades.

- Igualmente, el Plan Nacional de Evaluación de la calidad de las universidades.

Ante esta situación de relevancia valorativa hemos evaluado la Facultad de Ciencias Políticas y Sociología de la Universidad de Granada. Evaluación encaminada a conseguir los siguientes objetivos:

1. Negociar con todos los agentes de la Facultad, las dimensiones, las subdimensiones y los indicadores que van a ser evaluados.

2. Diseñar y elaborar los instrumentos necesarios para la evaluación.

3. Ensayar y validar, con carácter previo, los métodos, las técnicas y los instrumentos de evaluación a utilizar.

4. Conocer la realidad educativa de la Facultad, con la participación de todos los implicados en ella, para saber qué estamos haciendo.

\section{Cuestiones metodológicas}

La elección de un modelo u otro de evaluación no es una tarea fácil dado que supone adoptar una serie de decisiones metodológicas. Decisiones en las que intervienen múltiples factores como son nuestras concepciones ideológicas y filosóficas del mundo, nuestra peculiar forma de captar e interpretar los fenómenos y los hechos sociales o nuestra formación epistemológica, pero, como dice Fernández Sierra (1992), «sobre todo de la naturaleza del objeto de estudio y los objetivos que la evaluación se proponga».

Para ir clarificando los factores que intervienen en un proceso de evaluación empezaremos por determinar los criterios en los que se basan las decisiones metodológicas que vamos a tomar y que son la base del modelo que proponemos para evaluar la Facultad. Por parecernos la más adecuada para nuestro objeto de estudio, fundamentalmente seguiremos la propuesta reali- 
zada por Angulo Rasco y otros (1991), en su artículo «Evaluación educativa y participación democrática» ${ }^{1}$.

En primer lugar optamos por una evaluación que considera que las personas implicadas en el proceso de evaluación no son meros "objetos de estudio", sino sujetos activos en el desarrollo y la vida de la Facultad, estando implicados en todas las decisiones y valoraciones que presupone la evaluación, a la vez que se reconoce el pluralismo de valores e intereses diferentes.

Principios de actividad, pluralidad y diversidad que B. MacDonald (1985) recoge al considerar la Evaluación Democrática como uun servicio a la comunidad entera sobre las características del programa educativon; al igual que Beltrán (1991), en referencia directa a la evaluación de instituciones educativas - como en nuestro caso se trata-, la define como caquella en la que, tanto los métodos aplicados como la información generada en el proceso evaluador es abierta, y se discute y se reelabora entre los diferentes grupos de interés, formales o no, que configuran la comunidad educativa».

La negociación es otro de los criterios que debe inspirar todo proceso de evaluación, entendiendo por tal los diálogos y acuerdos que efectúan los evaluadores y los participantes de la institución a evaluar, antes, durante y después de la evaluación con el objetivo de evitar rechazos, reticencias, prejuicios y expectativas falsas de los distintos colectivos que integran la vida de una facultad o institución; en definitiva, aumentar la cultura evaluativa de la comunidad para evitar asociar evaluación con sanción y que nadie se pueda sentir amenazado o agredido en su independencia y autonomía. Pues, si no hay negociación, como apunta House (1979), "nadie desea ser evaluado".

1. Los autores defienden una evaluación externa que sirva a la sociedad y que cumpla las siguientes características:

- Ni la verdad ni la valoración correcta está en posesión de grupos o personas privilegiadas. La verdad y la valoración es asunto de construcción democrática de todos.

- No es el evaluador el que juzga, sino que facilita que sean los implicados los que lo hagan.

- No se pretende convertir las complejidades naturales en simplificaciones que falsifiquen la realidad.

- No pretende caer en la mística de los números o de las estadísticas.

- Se siente más comprometida con la utilización de procedimientos metodológicos sensibles a la riqueza, a la complejidad estructural y a las interacciones que se producen en toda realidad social.

- Parte de la clara explicitación de los criterios éticos que confirman los límites de su desarrollo.

- Tiene como objetivo material la publicidad de sus informes, y que rechazan cualquier imposición para mantenerlos en secreto o el ocultamiento de información valiosa.

- Cuidará que el lenguaje de sus informes sea accesible no sólo a los directamente implicados en la realidad evaluativa, sino también a cualquier otro ciudadano interesado.

- Pretende ser educativa.

- Reconoce que un informe de evaluación no es solamente un contenido que se hace público, sino que también puede crear una estructura social de relación y participación.

- Se siente comprometida con la sociedad en general y con el desarrollo y la profundización de la democracia política. 
La complejidad de los fenómenos sociales, y en este caso educativos, es de tal magnitud que al evaluar una institución educativa en la que intervienen multitud de factores, se puede correr el riesgo de seleccionar, explícita o tácitamente, las variables más fácilmente cuantificables u operativas, con el peligro de tomar en consideración los aspectos menos relevantes, los más nimios, los de menor interés educativo $\mathrm{y}$, por consecuencia, llegar a conclusiones sesgadas, erróneas y engañosas. En consecuencia tomamos como criterio la no simplificación de la complejidad natural para evitar que se falsifique la realidad de nuestro centro, utilizando métodos sensibles que capten la complejidad de los fenómenos evaluados. Como afirma Parlett y Hamilton (1987), la evaluación no intenta manipular, controlar o limitar las variables situacionales, sino abarcar toda la complejidad de la escena.

Partimos del principio de que el diseño o modelo de evaluación ha de ser emergente, flexible, siempre incompleto, siempre abierto a la evolución y al cambio según lo requieran las circunstancias y el desarrollo de la evaluación. El modelo que defendemos ha sufrido múltiples modificaciones para ir adaptándose a la realidad de la Facultad, así como a las diferentes opciones metodológicas que han tenido que tomarse durante el proceso de evaluación.

Los naturalistas, como afirma Guba (1985), al creer que se desenvuelven en un medio de realidades múltiples, entienden que la interacción con las personas investigadas modifica, con el tiempo, tanto a los investigadores como a ellos mismos, por lo que, ante la necesidad de teorías que toquen tierra, insistirán en un diseño abierto, emergente, que se despliega, se desarrolla y evoluciona en cascada, que nunca está completo hasta que la investigación se termina arbitrariamente cuando lo aconseja el tiempo, los resultados u otras consideraciones logísticas.

El modelo de la «figura» de Stake (1975) ha sido una de las bases teóricas en las que se apoya nuestro modelo, compartiendo con él su concepción de evaluación; en él destacamos dos actos básicos: la descripción, que engloba las intenciones, lo que se planea y se pretende, y las observaciones, lo que realmente se observa o se percibe. Otra operación principal es el juicio, referido a las normas utilizadas para emitir el juicio y/o los enjuiciamientos formulados sobre la institución o el programa evaluado. Otra idea que asumimos y que es introducida por Stake es la de la evaluación respondiente, llamada así porque debe responder a los problemas y las cuestiones reales que se puedan plantear a las personas implicadas en un programa o institución educativa. Entre las peculiaridades de este tipo de evaluación, Pérez Gómez $(1985)^{2}$ destaca el centrar-

2. Las otras peculiaridades presentadas por A. Pérez Gómez son:

- Enfatiza más los problemas que las teorías.

- Contempla las diferentes interpretaciones de las personas implicadas en el programa.

- Responde a las necesidades de información y al nivel de conocimiento de las personas interesadas en el programa.

- Enfatiza la necesidad de proporcionar a los interesados la experiencia del programa, implicándolos en su análisis.

- Describe y ofrece un retrato completo y holístico del programa. 
se más en la descripción de las actividades que en la definición de los objetivos o las intenciones de la institución.

Una vez presentados los diferentes criterios teóricos en los que nos hemos basado para elaborar el modelo de evaluación que posteriormente describimos, pasamos a señalar las distintas opciones metodológicas que acompañan a tales criterios. En primer lugar, hemos optado por una evaluación interna, entendiendo por tal aquella que realiza la propia comunidad y que tiene por finalidad el realizar una evaluación formativa con el objetivo de contribuir al perfeccionamiento de la realidad educativa del centro, tomando un enfoque global $u$ holístico, que considera la evaluación como un todo en el que todas las partes, todos los sujetos implicados, todas las fases de su desarrollo, son tenidas en cuenta de manera globalizadora.

Dentro de las perspectivas positivista/cuantitativa y naturalista/cualitativa, optamos por la complementariedad de ambos, superando tal disyuntiva y considerando que la mezcla de atributos de ambas perspectivas permite una mejora de la evaluación de los hechos sociales. Por último, entendemos que el estudio de casos al plantearlo en el estudio de las instituciones educativas como clarificación de su funcionamiento en una situación real contextualizada, respetando su especificidad institucional y con una finalidad descriptiva, es el que mejor se adapta a la perspectiva de la evaluación que se pretende.

\section{Descripción del modelo de evaluación y resultados}

En base a los planteamientos señalados, el modelo de evaluación seguido consta de tres niveles o fases: la primera denominada negociación; la segunda, descripción, y la tercera, valoración. Analicemos cada uno de ellos, señalando a la vez los resultados más relevantes después de su aplicación. En el primer nivel - negociación - todos los sectores de la comunidad universitaria, tanto profesores como alumnos y equipo directivo, determinan aquellas dimensiones que a su juicio son las más relevantes para la evaluación de la Facultad. A partir de ellas, se pueden establecer los criterios --según esta comunidad- que mejor definen la calidad de la enseñanza universitaria. En definitiva, se trataría de responder a la pregunta ¿qué evaluar?

La elección de dimensiones e indicadores se ha realizado mediante una propuesta inicial abierta, en la que aparecen toda una serie de hechos y acontecimientos que tienen lugar en esta comunidad universitaria, agrupados en seis bloques: Contexto, Recursos económicos y materiales, Profesorado, Alumnos, Organización y funcionamiento y Rectorado, y a partir de esta propuesta se han seleccionado las dimensiones y las subdimensiones objeto de evaluación.

Para conocer el posicionamiento de profesores, equipo directivo y alumnos, se ha utilizado como material e instrumentos de investigación un cuestionario de valoración de dimensiones y, como constatación del mismo, un grupo de discusión. En cada una de las 48 dimensiones de las que consta el cuestionario, se pide tanto a profesores como a alumnos dos aportaciones: una, que 
valoren la dimensión en relación con la relevancia que tiene para la evaluación de nuestra Facultad, en una escala que va del 1 al 5 , en donde el 1 significa "nada importante» y el 5, "muy importante»; la segunda aportación que se solicita consiste en contestar a una pregunta abierta sobre los aspectos a evaluar dentro de tal dimensión.

Una vez que ha finalizado tal proceso, se le plantean tres preguntas nuevas que hacen referencia a los seis bloques en los que se han agrupado las dimensiones, acerca de si sobran, faltan o consideran necesario que aparezcan los seis, y por último, deben señalar las aportaciones complementarias que consideren oportuno incluir para mejorar el proceso de evaluación de la Facultad.

Las conclusiones derivadas de la aplicación del primer nivel del modelo son:

1. Los seis bloques que inicialmente agrupaban las distintas dimensiones, van a permanecer según la ratificación realizada por la comunidad educativa de la Facultad, cuyo $81,7 \%$ considera que sí deberían aparecer los seis bloques, al igual que la información aportada por el grupo de discusión y el análisis factorial.

2. Las dimensiones que profesores y alumnos consideran que no deben incluirse en la evaluación de la Facultad son las siguientes:

- Relación y consideración de sus compañeros.

- Expectativas sobre el nivel de logro de los alumnos.

- Características personales de los alumnos.

- Características sociofamiliares.

- Antecedentes académicos.

- Elección de carrera.

- Reglamento de régimen interno.

- Administrador delegado.

- Conserjería.

3. De los aspectos a evaluar en cada dimensión, que denominamos subdimensiones o indicadores, hemos seleccionado aquellos que coinciden en ambos colectivos - profesores y alumnos.

El segundo nivel del modelo de evaluación, denominado descripción, corresponde a lo que sería la fase propia de la evaluación, siendo su finalidad la de describir la realidad de la Facultad por parte de todos los agentes que intervienen en ella - profesores, alumnos y equipo directivo- a partir de las dimensiones elegidas en el primer nivel, agrupadas en los bloques:

- Contexto.

- Recursos económicos y materiales.

- Profesorado.

- Alumnos.

- Organización y funcionamiento.

- Rectorado. 
Descripción que tiene por objetivo conocer la marcha de la Facultad, qué es lo que tiene, qué le falta y, en definitiva, qué se puede mejorar. Las técnicas utilizadas como instrumentos de recogida de información en la descripción de la Facultad son el Cuestionario de evaluación de la Facultad para profesores - CEFP-, Cuestionario de evaluación para alumno: - CEFA—, Cuestionario abierto para el equipo decanal - CAED -, Grupos de discusión y análisis de documentos.

Con la finalidad de conocer la estabilidad de los resultados en la evaluación de los profesores por parte de los alumnos, todos los profesores han sido evaluados por dos promociones de alumnos. De tal manera, que los profesores que imparten docencia en primero han sido evaluados por los alumnos de segundo y tercero, los que imparten docencia en segundo por los alumnos de tercero y cuarto, y así sucesivamente. De los resultados derivados de la aplicación del segundo nivel del modelo, por razones de espacio sólo analizamos las dimensiones "profesorado y alumnos", cabe destacar:

\section{De la dimensión "Profesorado"}

Al profesorado se le ha preguntado sobre su titulación académica, experiencia profesional y producción científica. Aproximadamente, la mitad de los profesores - $44 \%$ - son doctores, la distribución de los mismos, dentro del sector, es completa en catedráticos - 100\%-- para descender en titulares - $83 \%$-, asociados - 20\%-y ayudantes - $11 \%$ - Distribución obvia dentro de la carrera docente del profesorado universitario, ya que para ser titular o catedrático de Facultad es necesario ser doctor. Pero esto no quiere decir que el número de doctores sea el idóneo. Esta opinión coincide con el discurso dominante de profesores, alumnos y equipo decanal sobre el nivel de formación y preparación del profesorado.

En cuanto a formación didáctica, publicaciones, desarrollo de ponencias en congresos, conferencias impartidas, serninarios, pertenencia a grupos de investigación y estancia en centros de invest gación, nos centramos, en primer lugar, en las actividades desarrolladas a lo largo de toda la formación. Más de la mitad de los profesores dicen haber llevado a cabo las siguientes actividades:

a) Realización de cursos de formación pedagógica.

b) Publicación de libros, artículos - -en revistas nacionales-y capítulos de libros.

c) Presentación de ponencias.

d) Invitación a conferencias.

e) Organización de jornadas.

f) Participación en jornadas y reuniones científicas.

g) Y, por último, participación en investigaciones desarrolladas en su departamento.

Por el contrario, tan sólo el $20 \%$ ha publicado artículos en revistas internacionales, otro $38 \%$ ha permanecido en centros de investigación y un $22 \%$ ha participado en investigaciones a nivel internacional. La correlación entre la 
categoría profesional y las actividades investigadoras es estadísticamente significativa en las siguientes actividades:

Significación

- Formación pedagógica $0.014^{*}$

- Publicación de libros $\quad 0.000^{*}$

- Publicación de capítulos 0.023*

- Artículos en revistas internacionales $0.022^{*}$

- Invitado a dar conferencias $0.028^{*}$

- Participación en investigaciones a nivel nacional $0.032^{*}$

- Investigaciones a nivel internacional $0.004^{*}$

Por el contrario, las diferentes categorías de profesores llevan a cabo en porcentajes similares las siguientes actividades:

- Publicación de artículos en revistas nacionales.

- Presentación de ponencias.

- Organización de seminarios.

- Permanencia en centros de investigación.

- Pertenencia a grupos de investigación.

- Participación en investigaciones del departamento.

$\mathrm{O}$, lo que es lo mismo, ninguna de las cuatro categorías profesionales -ayudantes, asociados, titulares y catedráticos - presenta diferencias apreciables en la realización de dichas actividades. Probablemente porque las diferencias radiquen en la cuantía -número de artículos, etc. Al centrarnos en el trabajo desarrollado por los profesores en el último año, en cuatro de las seis actividades señaladas en el cuadro 1 más de la mitad de los profesores dicen haberla realizado. Por el contrario, tan sólo un $20 \%$ ha estado en centros de investigación y un $40 \%$ ha organizado conferencias o seminarios.

Al contrario que con la formación realizada a lo largo de toda la carrera, las actividades realizadas en el último año no correlacionan con la categoría pro-

Cuadro 1. Proporción de profesores que han realizado algunas de las actividades siguientes en el último año. (En porcentajes horizontales)

\begin{tabular}{lcrrr}
\hline Actividades & $\begin{array}{c}\text { Sí } \\
\%\end{array}$ & $\begin{array}{c}\text { No } \\
\%\end{array}$ & \multicolumn{1}{c}{$\begin{array}{c}\text { N/C } \\
\end{array}$} \\
\hline Publicación de libros, capítulos, artículos & 60 & 38 & 2 & 100 \\
Presentación de ponencias & 56 & 40 & 4 & 100 \\
Estancia en centros de investigación & 20 & 68 & 12 & 100 \\
Investigaciones terminadas o en marcha & 86 & 8 & 6 & 100 \\
Invitado a dar conferencias & & 70 & 30 & 100 \\
Organización de conferencias, cursos & 40 & 44 & 16 & 100 \\
(N) $(50)$ & & & & \\
\hline
\end{tabular}


fesional. Aun así, son los ayudantes y catedráticos los que presentan porcentajes más altos en la presentación de ponencias y estancias en centros de investigación. En la realización de investigaciones terminadas o en marcha son los ayudantes los que destacan.

En resumen, del análisis cuantitativo del currículo del profesorado, cabe destacar el perfil medio: doctor, tiene más de diez años de experiencia docente, lleva de tres a cuatro años en la Facultad, tiene dedicación plena a la enseñanza, posee formación didáctica, ha publicado libros, capítulos de libros y artículos en revistas nacionales, ha sido invitado a dar conferencias, ha organizado cursos, seminarios y jornadas y pertenece a grupos de investigación de su departamento.

Del análisis cualitativo destaca el escaso número de profesores y una formación menor de la deseada, demasiados intereses inmediatos - tesis, oposiciones-y pocos intereses intelectuales a largo plazo. Estas son algunas de las respuestas que marcan el discurso dorninante:

El nivel, tanto del profesorado como de los alumnos, me parece muy deficiente y encuentro que no hay mucho interés en mejorar esta situación (P596)

Su número es reducido, su formación, menor de la deseada como lo prueba el bajo número de doctores, con pocas estancias en centros de trabajo extranjero (ED1) ${ }^{3}$

El discurso dominante en los grupos de discusión se asemeja al anterior - aún más crítico--, sobre todo en lo referente a la formación y a la preparación del profesorado, se critica la falta de preparación suplantada, en ocasiones, por una buena voluntad. Voluntad, que ellos entienden - como alumnos-, que es interesada al ser la única preocupación del profesorado la consecución de la titularidad de la plaza.

$\mathrm{Al}$ ser una facultad nueva, muchos profesores, según los alumnos, "han encontrado un trabajo». Lo que ha provocado que la Facultad no cuente con profesores «famosos» de prestigio reconocido que a su vez formen a los nuevos y den un prestigio a la Facultad que marque de tal distinción a los profesionales que salgan de ella. Como esto no ha ocurrido, siguen diciendo los alumnos, sino todo lo contrario, la enseńanza es de mala calidad y los alumnos licenciados tendrán que hacer cursos de posgraduado. Comienza este discurso con la siguiente aseveración:

Yo creo que más que buena voluntad lo que hay es ganas de subsistir y de seguir aquí, y el paso siguiente es, pues, titularidad, tal, ocupa plaza. Además de que pueda haber una buena voluntad, pero... (2) B)

3. Hay otro discurso, minoritario, que califica la formación y la calidad del profesorado como:

- «Buena cualificación del profesorado» PA624

- "Por lo que se refiere a su cualificación, en general es alta aunque llama la atención que "áreas ajenas" a la Facultad "desbranen" a los profesores jóvenes en asignaturas de segundo ciclo o de una alta cualificación». ED2 
Cuadro 2. Media de la auroevaluación de los profesores.

\begin{tabular}{lrrrrrr}
\hline & \multicolumn{2}{c}{ Hombres } & \multicolumn{2}{c}{ Mujeres } & \multicolumn{2}{c}{ Profesores } \\
Total & \multicolumn{1}{c}{ X } & \multicolumn{1}{c}{$\mathrm{D}$} & \multicolumn{1}{c}{ X } & \multicolumn{1}{c}{$\mathrm{D}$} & \multicolumn{1}{c}{ X } & \multicolumn{1}{c}{$\mathrm{D}$} \\
\hline Primer ciclo & 68,3 & 6,8 & 68,6 & 4,9 & 67,7 & 10,6 \\
Políticas & 67,9 & 10,0 & 70,6 & 4,9 & 41,0 & 8,9 \\
Sociología & 69,1 & 6,2 & 68,4 & 4,6 & 71,2 & 10,7 \\
En ambas licenciaturas & 67,5 & 4,8 & 66,0 & 4,7 & 67,5 & 4,9 \\
Primero & 67,1 & 6,2 & 67,1 & 4,1 & 68,5 & 8,9 \\
Segundo & 77,2 & 4,4 & 70,4 & 3,7 & 75,3 & 5,1 \\
Tercero & 66,7 & 6,6 & 67,0 & 6,7 & 66,7 & 6,6 \\
Variables & & $\mathrm{Chi}^{2}$ & & G.L. & SIGN. & \\
\hline 1. Sexo & & 17,04 & & 18 & .51998 & \\
2. Categoría & & 59,91 & & 54 & .30035 & \\
3. Licenciatura & & 44,87 & & 36 & .14740 & \\
4. Satisfacción & & 125,69 & & 90 & $.00554^{*}$ & \\
5. Años en la docencia & & 87,07 & & 72 & .10880 & \\
\hline
\end{tabular}

Leyenda de las categorías utilizadas en cada variable: 1) Sexo: varones versus mujeres. 2) Categoría: ayudantes, asociados, titulares y catedráticos. 3) Licenciatura: Políticas versus Sociología. 4) Satisfacción: muy insatisfecho, bastante insatisfecho, algo insatisfecho, algo satisfecho, bastante satisfecho y muy satisfecho. 5) Años: menos de 3 , de 3 a 10 , de 11 a 20 , de 21 a 25 y más de 25.

No podemos olvidar que en el grupo de discusión hay un discurso soterrado, que identifica enseñanza de calidad con educación privada. «Educación pública", como ellos la llaman, enseñanza de mala calidad, e incluso defienden el incremento de las tasas universitarias para terminar con la masificación ${ }^{4}$.

\subsection{El quehacer docente}

En cuanto a los resultados del quehacer docente merece destacar, a nivel global, que el profesor se suele autoevaluar mejor de lo que lo hacen sus alumnos. Los profesores que mejor se autoevalúan son los que imparten docencia en Sociología $(X=69,1)$. En relación con el curso donde dan clase, son los de segundo los que se autoevalúan más alto. Por su parte, los alumnos de Políticas evalúan algo mejor a sus profesores que los de Sociología, al igual que hacen los de segundo en relación con primero y tercero.

Se constata que entre las autoevaluaciones de los profesores existen diferencias claras según el nivel de satisfacción profesional, siendo los más satisfechos los que mejor se autoevalúan, como queda reflejado en el cuadro 2.

4. 43.H- "Pero es que por muy buena que sea, si es pública, y si va a poder acceder a ella una gente que, bueno, que en teoría se supone que no se pueden permitir master por ahí, o lo que sea, que la formación que van a tener, formación pública, y que hay mucha gente de todas formas que por muy buena que fuese supongo que al final se volvería a encontrar lo mismo, mucha gente con la misma titulación, y que tendrían que seguir recurriendo a...». 
En relación con la evaluación del profesor por parte de los alumnos (cuadro 3), en términos generales, los alumnos que tienen más próxima la actuación del profesor - curso 91/92 - , evalúan mejor a los profesores; así se manifiesta en el $86 \%$ de los casos. El tiempo es, pues, una variable a controlar en la evaluación de los profesores por parte de los alumnos, a pesar de que las diferencias no son relevantes en todas las asignaturas.

La estabilidad de los resultados --causa de evaluar a cada profesor dos veces-, en términos generales, se confirma. Los profesores que son evaluados con puntuaciones extremas - "mejores y peores" - permanecen en ambas evaluaciones - 1C.2, 2F, 2I, 3B.1, 3H-, lo que constata la fiabilidad del cuestionario y de los resultados. Asimismo, no existen diferencias apreciables en la valoración realizada por los alumnos por razón del sexo del profesor.

$\mathrm{La}$ influencia de las variables contextuales y personales de los alumnos sobre la evaluación del profesor, todas la variables, tanto las contextuales como las personales, actúan como diferenciadores en los alumnos de segundo y tercero a la hora de evaluar a los profesores de primero. En otras palabras, influyen en la evaluación que hacen de los mismos. La variable satisfacción señala diferencias notables en todas las asignaturas, frente al sexso y la asistencia, que sólo lo hace en tres.

En las asignaturas de primero hay inna marcada diferencia al incidir menos variables en la evaluación de profesores que imparten las asignaturas de segundo.

Cuadro 3. Media de la evaluación de los profesores por parte de las dos promociones de alumnos y profesores.

\begin{tabular}{|c|c|c|c|c|c|}
\hline \multirow[b]{2}{*}{$\begin{array}{l}\text { Profesores } \\
\text { de primero: } 1^{*}\end{array}$} & \multicolumn{2}{|c|}{ Curso 90/91 } & \multicolumn{2}{|c|}{ Curso 91/92 } & \multirow[b]{2}{*}{$\begin{array}{c}\text { Profesor } \\
\text { X }\end{array}$} \\
\hline & \multicolumn{2}{|c|}{ Alumnos de tercero } & \multicolumn{2}{|c|}{ Alumnos de segundo } & \\
\hline $1 \mathrm{~A} .1$ & 56.6 & 11.7 & 62.1 & 11.9 & 64.0 \\
\hline $1 A .2$ & 53.0 & 11.2 & 72.0 & & \\
\hline $1 \mathrm{~A} .3$ & 50.8 & 10.9 & 58.7 & 11.2 & 66.0 \\
\hline $1 \mathrm{~A} .4$ & 57.7 & 12.1 & 55.6 & 11.0 & \\
\hline $1 A .5$ & 53.5 & 9.6 & 67.0 & & \\
\hline 1B.1 & 65.6 & 11.1 & 71.0 & & \\
\hline $1 \mathrm{~B} .2$ & 57.5 & 11.7 & 57.5 & 12.1 & 78.0 \\
\hline 1B.3 & 52.1 & 13.0 & 56.8 & 11.6 & 72.0 \\
\hline $1 \mathrm{C.1}$ & 47.1 & 10.9 & 53.1 & 11.8 & 72.0 \\
\hline $1 C .2$ & 39.4 & 9.9 & 45.6 & 10.2 & \\
\hline 1D.I & 57.0 & 12.7 & 57.0 & & \\
\hline $1 \mathrm{D} .2$ & 50.0 & 13.2 & 52.6 & 12.0 & 62.0 \\
\hline $1 \mathrm{D} .3$ & 49.5 & 11.7 & & & \\
\hline $1 \mathrm{E} .1$ & 50.6 & 12.8 & 51.3 & 13.2 & 66.0 \\
\hline 1E. 2 & 49.7 & 13.5 & 56.7 & 12.6 & \\
\hline $1 F .1$ & 64.3 & 12.4 & 62.7 & 11.5 & 66.0 \\
\hline 1F. 2 & 59.4 & 10.9 & 52.1 & 10.3 & 61.0 \\
\hline 1F.3 & 51.2 & 14.1 & 60.3 & 10.7 & \\
\hline 1F.4 & 58.1 & 11.2 & 62.0 & & \\
\hline
\end{tabular}


Cuadro 3. (Continuación)

\begin{tabular}{|c|c|c|c|c|c|}
\hline \multirow{2}{*}{$\begin{array}{l}\text { Profesores } \\
\text { de segundo: } 2^{*}\end{array}$} & \multicolumn{2}{|c|}{ Curso 90/91 } & \multicolumn{2}{|c|}{ Curso $91 / 92$} & \multirow[b]{2}{*}{$\begin{array}{c}\text { Profesor } \\
\quad \mathrm{X}\end{array}$} \\
\hline & \multicolumn{2}{|c|}{$\underset{X}{\text { Alumnos de cuarto }}$} & \multicolumn{2}{|c|}{ Alumnos de tercero } & \\
\hline $2 A .1$ & 47.9 & 13.4 & 74.0 & & \\
\hline 2A. 2 & 63.8 & 14.3 & 71.0 & & \\
\hline $2 A .3$ & 64.6 & 12.6 & 71.0 & & \\
\hline 2B. 1 & 52.3 & 11.1 & 60.6 & 11.6 & 67.0 \\
\hline 2B. 2 & 55.0 & 11.3 & 55.4 & 11.9 & 72.0 \\
\hline 2C. 1 & 66.6 & 10.9 & 72.0 & & \\
\hline $2 C .2$ & 60.9 & 12.2 & 65.4 & 11.5 & \\
\hline $2 \mathrm{D}$ & 50.4 & 16.5 & 59.7 & 11.8 & 74.0 \\
\hline $2 \mathrm{E}$ & 50.1 & 11.3 & 56.6 & 13.8 & 71.0 \\
\hline $2 \mathrm{~F}$ & 61.2 & 12.9 & 62.7 & 12.9 & 74.0 \\
\hline $2 \mathrm{G}$ & 52.2 & 15.9 & 64.0 & 12.2 & 81.0 \\
\hline $2 \mathrm{H} .1$ & 54.8 & 12.5 & 70.0 & & \\
\hline $2 \mathrm{H} .2$ & 48.5 & 15.7 & 55.1 & 13.0 & 67.0 \\
\hline $2 \mathrm{I}$ & 35.7 & 10.1 & 39.7 & 12.6 & 72.0 \\
\hline $\begin{array}{l}\text { Profesores } \\
\text { de tercero: } 3^{*}\end{array}$ & $\underset{X}{\text { Alumr }}$ & quinto & $\underset{X}{\text { Alum }}$ & $\begin{array}{l}\text { cuarto } \\
\text { D }\end{array}$ & $\begin{array}{c}\text { Profesor } \\
\quad \mathrm{X}\end{array}$ \\
\hline $3 \mathrm{~A}$ & 58.3 & 11.2 & 60.8 & 11.6 & \\
\hline 3B. 1 & 44.2 & 9.9 & 49.6 & 12.2 & 67.0 \\
\hline 3В. 2 & 42.7 & 10.0 & 51.6 & 13.3 & 77.0 \\
\hline $3 B .3$ & 48.8 & 9.8 & 56.5 & 13.9 & 55.0 \\
\hline $3 C .1$ & 47.1 & 11.6 & 72.0 & & \\
\hline 3 C. 2 & 49.3 & 11.4 & 61.0 & & \\
\hline 3 C. 3 & 42.5 & 10.2 & 69.0 & & \\
\hline $3 \mathrm{D}$ & 51.5 & 12.7 & 53.6 & 11.8 & 76.0 \\
\hline $3 \mathrm{E}$ & 63.0 & 7.9 & 50.7 & 10.4 & \\
\hline $3 \mathrm{~F} .1$ & 56.5 & 9.7 & & & \\
\hline $3 F .2$ & 56.9 & 11.8 & 67.0 & & \\
\hline $3 G$ & 61.1 & 11.1 & 58.7 & 9.3 & 66.0 \\
\hline $3 \mathrm{H}$ & 65.0 & 9.2 & 64.5 & 8.1 & 69.0 \\
\hline $3 I$ & 50.8 & 13.7 & 59.4 & 12.0 & 71.0 \\
\hline $3 \mathrm{~J}$ & 45.4 & 12.0 & 57.0 & & \\
\hline
\end{tabular}

$1^{*}$ Alumnos matriculados en el curso $92 / 93$ en tercero y segundo, respectivamente.

$2^{*}$ Alumnos matriculados en el curso $92 / 93$ en cuarto y tercero.

$3^{*}$ Alumnos matriculados en el curso $92 / 93$ en quinto y cuarto. 
Es decir, que en la evaluación realizada por los alumnos de tercero y cuarto curso 92/93 - a los profesores de segundo, la influencia ejercida por las variables contextuales y personales es menor que la efectuada en primero. Hecho que da más fiabilidad a la evaluación al suponer que se debe al quehacer del profesor y no a otras variables ajenas al proceso.

De la evaluación de los profesores de tercero destaca el reducido número de variables que presenta una diferencia estadísticamente significativa con la evaluación realizada por los alumnos de cuarto y quinto. Esto indica un alto índice de frabilidad en la evaluación y constata de nuevo que los alumnos de cursos más avanzados constituyen una fuente de información más fiable en la evaluación de los profesores.

Nos resta para finalizar con el análisis de la evaluación del profesor por parte de los alumnos y la realizada por los propios profesores - autoevaluación-, descifrar en qué aspectos de ésta coinciden y en cuáles no. Para ello, hemos dividido las preguntas, referentes al quehacer docente, en cuatro bloques:

1. Cumplimiento de la labor docente.

2. Programa de la asignatura.

3. Desarrollo de las clases.

4. Relación con los alumnos.

En cada uno de estos bloques se ha examinado el grado de acuerdo entre profesor y alumnos. De este modo, hemos extraído el perfil medio de profesor, según el nivel de acuerdo y la tendencia de las respuestas, que imparte docencia en cada uno de los cursos. Así, el profesor que imparte docencia en primero y tercero, se caracteriza por ser accesible, cooperante, manifiesta interés por los alumnos y además se nota satisfecho con su trabajo. Por el contrario, no utiliza la metodología adecuada en clase, no suele atender las iniciativas de los alumnos, no es buen didacta, no utiliza el tipo de evaluación adecuado y tampoco apoya las explicaciones con recursos tecnológico-didácticos.

Mientras que el profesor que da clase en segundo destaca por cumplir los horarios de clase y tutoría, realizar las prácticas de la asignatura, revisar los exámenes y cumplir el programa de la asignatura. Sin embargo, no utiliza la metodología adecuada en clase, no suele atender las iniciativas de los alumnos, no es buen didacta, no utiliza el tipo de evaluación adecuada y tampoco apoya las explicaciones con recursos tecnológico-didácticos.

A conclusiones similares responde el discurso dominante en los grupos de discusión, que es crítico con el profesorado. Este discurso diseña un perfil de profesor caracterizado. En primer lugar, por la falta de experiencia, planificación y preparación, sobre todo, de formación didáctica. Desarrolla un tipo de clase «tradicional», teórica y poco participariva, y, como dicen los alumnos, «sueita el rollo y desaparecen:

La mayoría de los profesores, sobre todo por los años que llevamos aquí, los que llevan años aquí, no dan nada, recogen las fichas, los que se suben a la tarima, y critican con que antes, cuando ellos estudiaban, el profesor se ponía su atril y soltaba el rollo y resulta que están haciendo lo mismo (B59). 
Por otro lado, los alumnos consideran que los programas de las asignaturas son muy teóricos, alejados de la realidad social, al mismo tiempo, se solapan unos con otros al no coordinarse los profesores:

Aquí se teoriza sobre todo, pero aquí aplicado nada, eso vamos. Cuando este año, en quinto, nos han dicho que nos van a dar Psicología Social aplicada, nos hemos puesto todos «la mar» de contentos.

Otra de las características del profesor, según los alumnos, es la escasa o nula autonomía dada a los estudiantes en el proceso de enseñanza-aprendizaje. Se obliga a todos los alumnos a hacer las mismas lecturas, trabajos, exámenes..., sin permitir la más mínima iniciativa o tolerar otra visión distinta de la suya. Los ejemplos quizás sean más elocuentes que cualquier descripción:

[...] hablando del profesor, ¿Qué capacidad de trabajo en grupo se puede llegar a adquirir? ¿Qué capacidad de trabajo paralelo, a lo que es la disciplina de exámenes y de profesores dentro del aula? ¿Puede adquirir un grupo de alumnos autonomía? ¿Qué grado de desviación puede tener respecto a lo que son los exámenes y lo que es la enseñanza de un programa? ¿Qué capacidad de crear nuevas bibliografías autónomas de esos alumnos, de relacionarse con otra gente, de enlazar con otras facultades se puede llegar a tener? (B87).

Opinión compartida por el profesor I. Sotelo $(1993)^{5}$, al criticar el hecho de que la universidad española se limita a divulgar curso tras curso una introducción a cada una de las ciencias, que es lo que luego exige en los exámenes, unas pruebas "múltiples y tan próximas temporalmente unas de otras que impiden a los alumnos centrarse en el tema científico que realmente les interesa». Parafraseando a Unamuno, dice Sotelo, "si encuentra ese tema, el alumno está perdido, ya que su labor se reduce a salvar una carrera de obstáculos. Los mejores estudiantes suelen ser los peores, ya que o se tragan examen tras examen o se dedican a aprender sólo lo que realmente les interesa y descuidan lo demás".

Por último, seriedad y responsabilidad, no son cualidades del profesorado de la Facultad; según este discurso dominante, los cambios de clases, los exámenes, los horarios de tutorías y las clases de prácticas se modifican o se suprimen según los intereses y las necesidades de los profesores, sin consultar o mirar por los intereses de los alumnos:

Sí, bueno, la costumbre de las tutorías en el horario en que tu tienes clase es general. Si tu tienes clase, como nosotros, desde las ocho y media a las dos y media un par de días a la semana, pues descuida que todas las tutorías por la tarde. Entonces han dicho, uno que la ha puesto dice: «mira es que yo os estoy haciendo un favor", dice: «yo podría ponerlas por la mañana, porque a mí nadie me obliga a ponerlas por la tarde».

5. Suplemento de educación de El País, martes 2 de febrero de 1993. 


\subsection{La identificación con el trabajo}

En general, los profesores están más satisfechos que insatisfechos con su trabajo; tan sólo un $12 \%$ declara estar algo insatisfecho, el $4 \%$ bastante insatisfecho, y el $32 \%$ poco satisfecho - cuadro 4 . Las diferencias entre categorías de profesores no son notables. Aun así, los catedráticos son los más satisfechos, luego los asociados y titulares y por último los ayudantes. Las diferencias por sexo tampoco son apreciables.

Respecto a las causas de la satisfacción, destaca el reconocimiento de los profesores de que les gusta el trabajo que realizan -26\%-. El porcentaje de los que indican este motivo es más reducido entre catedráticos y titulares, señalando como motivo otras razones no especificadas.

Las causas de la insatisfacción son variadas. De éstas destacan las siguientes:

- La competitividad entre compañeros, $22 \%$.

- Los alumnos, $11 \%$

- La necesidad de actualización, 11\%.

\section{De la dimensión "Alumnos"}

No es una novedad si decimos que las condiciones, los hábitos y las formas de estudio de los alumnos coadyuvan a los resultados finales de los estudiantes. Ante tal premisa, analizamos la valoración realizada por los alumnos sobre tales hechos. En líneas generales, los alumnos de la Facultad consideran buenas las condiciones de su lugar habitual de estudio, así lo manifiestan más de las tres cuartas partes de los alumnos - $24 \%$ muy buenas, $45 \%$ bastante buenas, $18 \%$ algo buenas-, el 7\% piensa que son algo malas y tan sólo el 3,2\% las encuentra bastante malas o muy malas.

En cuanto a los hábitos de estudios, la mitad de los alumnos confiesan no estudiar con regularidad. De éstos el 19\% lo hace ocasionalmente, un 15\% cuando le apetece y el $14 \%$ para los exámenes - cuadro 5-. Al centrarnos

Cuadro 4. Distribución de profesores según categoría profesional en relación con el grado de satisfacción con su trabajo.

\begin{tabular}{lrrrrrr}
\hline & $\begin{array}{l}\text { Total } \\
\text { opiniones \% }\end{array}$ & $\begin{array}{l}\text { Ayudantes } \\
\%\end{array}$ & $\begin{array}{l}\text { Asociados } \\
\%\end{array}$ & $\begin{array}{l}\text { Titulares } \\
\%\end{array}$ & $\begin{array}{l}\text { Catedráticos } \\
\%\end{array}$ \\
\hline Muy insatisfecho & 4 & 11 & 6 & & \\
Bastante insatisfecho & 4 & 11 & & & \\
Algo insatisfecho & 12 & 11 & 10 & 17 & \\
Algo satisfecho & 16 & 33 & 20 & 13 & \\
Bastante satisfecho & 56 & 44 & 60 & 56 & 67 \\
Muy satisfecho & 8 & 10 & 6 & 33 & \\
\hline Total (N) & $(50) 100$ & (9) 100 & (20) 100 & (18) 100 & (3) 100 \\
\hline
\end{tabular}


Cuadro 5. Proporción de alumnos según forma y hábitos de estudio.

\begin{tabular}{|c|c|c|c|c|c|}
\hline & $\begin{array}{l}\text { Total } \\
\text { hábitos \% }\end{array}$ & $\begin{array}{l}\text { Segundo } \\
\%\end{array}$ & $\begin{array}{l}\text { Tercero } \\
\%\end{array}$ & $\begin{array}{l}\text { Cuarto } \\
\%\end{array}$ & $\begin{array}{l}\text { Quinto } \\
\%\end{array}$ \\
\hline Regularmente & 50 & 46 & 55 & 50 & 53 \\
\hline Ocasionalmente & 19 & 25 & 19 & 14 & 11 \\
\hline Cuando me apetece & 15 & 14 & 11 & 15 & 22 \\
\hline Para los exámenes & 14 & 14 & 12 & 17 & 12 \\
\hline Nunca & 0.3 & 0.6 & 0.9 & & \\
\hline $\mathrm{N} / \mathrm{C}$ & 0.6 & 1 & 2 & & \\
\hline Total (N) & (629) 100 & (255) 100 & (178) 100 & (114) 100 & (82) 100 \\
\hline
\end{tabular}

en las diferencias por curso, observamos que los alumnos de los últimos cursos tienden a estudiar regularmente, frente a los de los primeros que lo hacen en ocasiones. Los que manifiestan estudiar más y menos horas son los alumnos de los primeros cursos, sobre todo los de segundo, que presentan la distribución más homogénea dentro de la escala. Por el contrario, los alumnos de los últimos cursos tienen una marcada tendencia hacia posiciones medias de la escala. El mayor porcentaje de alumnos de cuarto y quinto manifiesta estudiar entre 11 y 15 horas, frente al $9 \%$ y al $5 \%$ que declara estudiar más de 20 horas.

En relación con el número de clases a que asisten los alumnos, aproximadamente algo más de la mitad afirma asistir a todas - $63 \%$ - La otra mitad, se distribuye en torno a las 8 y 15 clases semanales - $12 \%$ y $26 \%$ respectivamente-, lo que supone una media de 2 y 4 horas al día. No existen diferencias apreciables por razón del curso, si bien se aprecia, en los alumnos de quinto, una tendencia positiva hacia la asistencia, frente a los de segundo, tercero y cuarto en que suele ser algo menor.

Si entre los alumnos de los diferentes cursos no existen diferencias en cuanto a las condiciones de lugar de estudio, hábitos, dedicación y asistencia a clase, por el contrario, entre alumnos y profesores existen diferencias significativas en cuanto a la percepción del número de alumnos que asisten habitualmente a clase. El $88 \%$ de los profesores opinan que asisten asiduamente a clase entre 40 y 100 alumnos, mientras el $86 \%$ de los alumnos consideran estas cifras algo menores de, 21 a 80 - cuadro 6.

A la vista de los resultados podemos concluir que no hay un único perfil del alumno, sino como mínimo dos. Uno que podríamos llamar «modélico", caracterizado por tener un lugar agradable de estudio, asistir a todas las clases y estudiar regularmente. Por el contrario, el otro perfil se caracteriza por tener un lugar de estudio regular, asistir a algunas clases y estudiar ocasionalmente o cuando le apetece.

\subsection{Los resultados académicos}

El estudio de los resultados académicos es amplio y complejo por sí sólo; así, ha 
Cuadro 6. Proporción de alumnos que asisten a clase con regularidad según profesores y alumnos.

\begin{tabular}{lccc}
\hline & Total cantidad \% & Profesores \% & Alumnos \% \\
\hline Menos de 20 & 2 & 2 & \\
De 21 a 40 & 22 & 9 & 23 \\
De 41 a 60 & 33 & 32 & 33 \\
De 61 a 80 & 30 & 34 & 30 \\
De 81 a 100 & 10 & 22 & 9 \\
Más de 100 & 2 & 2 & 2 \\
No contesta & 3 & 10 & 2 \\
\hline Total (N) & (679) 100 & (50) 100 & (629) 100 \\
\hline
\end{tabular}

sido el objeto de estudio de tesis e investigaciones ${ }^{6}$. Nosotros nos centraremos en los resultados académicos referidos a los cursos $90 / 91$ y $91 / 92$ en la convocatoria de junio, haciendo especial hincapié en los alumnos repetidores y en las variables que diferencian unos alumnos de otros. Cabe destacar el descenso de aprobados y no presentados al avanzar curso, frente al aumento de aprobados. De éstos, hay una tendencia a valores más positivos -notables y sobresalientes- Estos resultados no difieren de los de otras facultades similares del Estado español ${ }^{7}$.

La diferencia entre las asignaturas que más suspensos concentran y las que menos es notable - 25 puntos-. Diferencias que no se reproducen en ninguna de las otras calificaciones - aprobado, notable, sobresaliente--. En cuanto a los repetidores, en la distribución por sexo, los hombres repiten más que las mujeres en todos los cursos. El 15\% de los hombres de primero son repetidores, frente al $11,1 \%$ de mujeres. En segundo, el porcentaje es el 10,9\% de los hombres y el 9,5\% de las mujeres, en tercero, el 20,2\% de los hombres matriculados repiten curso, aunque tan sólo el 13\% de las mujeres lo hacen. A conclusiones parecidas han llegado los estudios realizados por M. Latiesa (1991), L. Salvador y A. García-Valcárcel (1989), y R. González Tirados (1989).

Del estudio de las variables que inciden en los resultados de los alumnos, podemos deducir algunas conclusiones. En conjunto, son pocas las variables que marcan diferencias significativas. Al igual que en la evaluación del profesor por parte de los alumnos, en los cursos más bajos influyen más variables que en los últimos - tercero.

6. Entre otros cabe citar a:

- LATIESA, M. (1991): El rendimiento académico en distintos paises y centros universitarios.

- MARÍN IBÁŃNEZ (1991): El rendimiento de la Universidad.

- Salvador, L.; García, A. (1991): El rendimiento académico en la Universidad de Cantabria: Abandonos y retrasos en los estudios.

- GonZÁlez TIRADOS (1992): Principales dificultades en el rendimiento académico en primer año de carrera de Ingeniería.

7. Véase LAURENTINO, S. (1989): El rendimiento académico en la Universidad de Cantabria. 
Las variables personales - sexo, edad, asistencia, hábitos, satisfacción-son las que actúan como mayores diferenciadores en los resultados de los alumnos. Las contextuales -licenciatura-, tan sólo afectan a tres asignatura del total -Introducción a la Ciencia Política e Historia de primero y Estructura Social Contemporánea de tercero-, siendo mejores los resultados de los alumnos de Sociología y más negativos los de Políticas. El sexo es de las variables que producen diferencias apreciables en las notas de los alumnos, en el mayor número de asignaturas, siendo los alumnos los que obtienen mejores notas que las alumnas, al contrario de lo que ocurría en el porcentaje de repetidores ${ }^{8}$.

En relación con los hábitos de estudio de los alumnos, se aprecian diferencias significativas en algunas asignaturas, sobre todo en las de primero, al ser los alumnos que estudian con mayor regularidad los que obtienen mejores calificaciones ${ }^{9}$. La edad de los estudiantes es otra de las variables, junto con el sexo, que en asignaturas de primero y segundo señala diferencias importantes; se aprecia una marcada tendencia positiva a medida que aumenta la edad del alumno. $\mathrm{O}$, lo que es lo mismo, los alumnos de mayor edad obtienen mejores notas que los más jóvenes ${ }^{10}$.

8. Según L. García Aretio (1991), son mayoría los estudios que, referidos a la Universidad, confirman que no existen diferencias significativas de rendimiento debidas al sexo de los individuos. Así se deduce de los trabajos de carácter nacional e internacional, tales como los de May y otros (1977), Herrero e Infestas (1980), Carney y Geys (1981), L. Salvador (1989) y Latiesa (1992), este último referido al abandono. Por el contrario, algunas diferencias detectaron Weitz y Colver (1958) y Hood (1957). Asimismo, García Llamas (1986) encontró que en la UNED y en la carrera de Ciencias de la Educación, el rendimiento académico de los varones es significativamente más alto que el de las mujeres.

9. La nota de los exámenes parciales y la preparación diaria, son variables que inciden en el rendimiento de los alumnos de la Universidad Politécnica de Madrid, según el estudio llevado a cabo por R. González Tirados (1989).

10. En lo que respecta a la edad, Patton (1958) concluye que los universitarios cuanto más jóvenes ingresan, tienen más probabilidad de éxito. Sin embargo, si se retrasa la entrada, la probabilidad de éxito será más elevada cuanto más edad tenga el estudiante. En los estudios referidos a la Universidad, no se han encontrado generalmente diferencias significativas en el rendimiento relacionadas con la edad. De ello pueden dar muestra los trabajos de McDonald y McPherson (1975), Wileman y otros (1981), L. García Aretio (1991).

El estudio llevado a cabo por J.L. García Llamas (1086), descubre que existe relación significativa entre la edad y el rendimiento de los alumnos de la UNED, deduciendo que el grupo más numeroso de alumnos - los de 23 a 29 años-, que en principio parece una edad óptima para el estudio, es el que en mayor grado opta por no presentarse a examen, siendo sus rendimientos bajos o medios. Por su parte, M. Latiesa (1992), encontró que los alumnos de más edad abandonan, fracasan, en mayor medida en los estudios universitarios. También, Infestas (1986), en investigación realizada en la Universidad de Salamanca, encontró que los mayores de 25 años abandonaban la Universidad en mayor medida que los más jóvenes. En lo que respecta a la Universidad de Cantabria, L. Salvador y otros (1989) descubren que la forma de ingreso y la edad son las variables que presentan mayor asociación con el rendimiento académico, siendo los más jóvenes, aquéllos que ingresan con menos de 20 años e inician los estudios superiores como continuación de los medios a través del $\mathrm{COU}$, los que presentan un mejor rendimiento. 


\subsection{La identificación con los estudios}

Los estudiantes que acceden a la educación superior están condicionados en su elección por determinadas variables, especialmente por la oferta de plazas y las trayectorias académicas anteriores, M. Latiesa (1992). Estas variables influyen, con independencia de que los alumnos sean conscientes de ellas o no, por el contrario, hay otras variables - subjetivas- que los estudiantes manifiestan que han determinado su elección y que nosotros hemos intentado determinar.

Estas variables que hemos denominado subjetivas, ya que obedecen a manifestaciones conscientes de los alumnos, han sido, por grado de influencia, las siguientes:

10. Una vocación personal, me gusta $-80 \%$.

$2^{\circ}$. Me gusta la profesión para la que me capacita $-78 \%$.

$3^{\circ}$. Obtener una cultura general $-77 \%$.

$4^{\circ}$. Necesidad de matricularme en alguna facultad $-18 \%$.

$5^{\circ}$. No me han admitido en otra $-11 \%$.

$6^{\circ}$. Por no trasladarme a otra ciudad distinta de donde resido $-10 \%$.

$7^{\circ}$. Trasladarme a otra ciudad distinta de donde resido $-8 \%$.

Como podemos observar, hay una opinión mayoritaria sobre la elección vocacional o profesional, siendo una minoría la influida por las variables coyunturales o culturales. De todos modos, de las variables seńaladas por los alumnos como influyentes, hay tres de ellas que no afectan por igual a todos. «La necesidad de matricularse en alguna facultad» es una de ellas, al presentar diferencias significativas en la distribución por curso, los alumnos de los últimos cursos tienden a justificar su opción por este motivo.

"La obtención de una cultura general» y "el poder trasladarse a otra ciudad», presentan diferencias notables entre los alumnos, siendo, de nuevo, los alumnos de cuarto y quinto los que tienden a esto con mayor frecuencia. Por tanto, podemos deducir, con todas las reservas de poder equivocarnos, que a los alumnos, de las dos primeras promociones de la Facultad les influyeron a la hora de elegir la carrera más las variables personales que las estrictamente profesionales.

Es una hipótesis defendida, también, por los alumnos que intervienen en los dos grupos de discusión, al entender que muchos alumnos de la Facultad son de «rebote». O, lo que es lo mismo, los alumnos optaron por esta carrera, bien porque no les admitieron en otra $-11 \%$, porque tenían que estudiar algo o, simplemente, porque les permitía trasladarse a otra ciudad:

La mayoría de la gente viene de $3^{\circ}$ rebote y existe la idea de que esta es una Facultad de cachondeo (B106F).

En cuanto a la satisfacción o a la insatisfacción del alumnado con su trabajo de estudiante, en general, los alumnos están más satisfechos que insatisfechos con sus estudios; tan sólo un 3\% declara estar muy insatisfecho, el $8 \%$ bastante insatisfecho y el $11 \%$ algo satisfecho - cuadro 7 - Las diferencias 
Cuadro 7. Satisfacción por los estudios, según curso matriculado.

\begin{tabular}{lcrrrrr} 
& $\begin{array}{l}\text { Total } \\
\text { satisfacción \% }\end{array}$ & $\begin{array}{l}\text { Segundo } \\
\%\end{array}$ & $\begin{array}{l}\text { Tercero } \\
\%\end{array}$ & $\begin{array}{l}\text { Cuarto } \\
\%\end{array}$ & \multicolumn{2}{c}{$\begin{array}{l}\text { Quinto } \\
\%\end{array}$} \\
\hline Muy insatisfecho & 3 & 3 & 3 & 1 & 3 \\
Bastante insatisfecho & 8 & 5 & 8 & 12 & 15 \\
Algo insatisfecho & 11 & 7 & 13 & 12 & 18 \\
Algo satisfecho & 16 & 16 & 11 & 20 & 18 \\
Bastante satisfecho & 46 & 50 & 46 & 47 & 39 \\
Muy satisfecho & 14 & 18 & 17 & 6 & 5 \\
N/S & & & & & & \\
N/C & $(629) 100$ & $(255) 100$ & $(178) 100$ & $(114) 100$ & $(82) 100$ \\
\hline Total (N) & & & & &
\end{tabular}

entre cursos son notables. Quiere esto decir que la asociación entre cursos de pertenencia y grado de satisfacción es alta. Así, los alumnos de quinto son los menos satisfechos seguidos de los de cuarto y tercero, y por último los más satisfechos son los de segundo.

Respecto a las causas de la satisfacción, destaca el reconocimiento de los alumnos de que les gustan los estudios que realizan - $40 \%$ - El porcentaje de los que indican este motivo es más reducido entre los alumnos de tercero, $y$ señalan como motivo la formación que dan, siendo éste, a su vez, el segundo de los motivos que mencionan los alumnos - $24 \%$ - seguido de los contenidos $--23 \%$ - Entre las causas de la insatisfacción destaca el plan de estudios $-53 \%$, las pocas salidas profesionales - 15\%-, los profesores - $12 \%$ - los resultados académicos - $10 \%$ - y por último la elección de otra carrera - 3\%.

Para finalizar, sólo nos queda analizar la última fase de la evaluación, que tiene lugar en el nivel tercero del modelo denominado valoración, en el que todos los agentes de la comunidad hacen un juicio de valor sobre la Facultad. En esta línea hemos propuesto a profesores y alumnos que evalúen la Facultad en su conjunto en una escala entre 1 y 10 , donde el 1 corresponde a la valo-. ración negativa y el 10 a la positiva, al mismo tiempo que indicasen las razones por las cuales otorgaban una u otra valoración. Pues bien, la puntuación más frecuente es la otorgada al 4: el $47 \%$ del total de la muestra opta por valores negativos. $\mathrm{O}$ sea, concede un suspenso a esa evaluación global de la Facultad. Respuesta que, por grupos, presenta diferencias significativas, siendo los profesores los que evalúan más positivamente a la Facultad. Es decir, los alumnos tienden a valorar negativamente la Facultad, mientras los profesores lo hacen a la inversa.

Las razones alegadas a la hora de justificar la evaluación son muchas, y son diferentes, también, entre los colectivos. Entre los alumnos señalan por frecuencia las siguientes: 
1. Profesorado

2. Instalaciones

3. Compartir edificio con otras licenciaturas

4. Información

5. Plan de estudios

6. Masificación

7. Participación

8. Motivación

Por el contrario, los profesores sefialan otra serie de aspectos o acciones para justificar la evaluación de la Facultad. Entre ellas se imponen:

1. Masificación

2. Preparación previa de los alumnos

3. Relación de la Facultad con el medio

4. Investigación

5. Metodología

6. Organización y funcionamiento

7. Plan de estudios

8. Departamentos

\section{Bibliografía}

ÁLvarez, C. (1989). «Evaluación de la docencia en la UAM». Boletín del Instituto de Ciencias de la Educación, 14.

Angulo, F.; Fernández Sánchez, J.; Martínez ARIAS, M R. (1987). Cuestionario de evaluación del profesorado de la Universidad Complutense. Madrid: ICEUM. (Texto policopiado).

Angugo Rasco, J.F; Contreras Domingo J. y Santos Guerra (1991). «Evaluación educativa y participación democrática». Cuadernos de Pedagogia, núm. 195, septiembre. Barcelona.

APEZECHEA, H. (1989). «Modelos de investigación, diseño de prueba e investigación cualitativa». Punto, 21, 47/48, (17-21).

Beltrán, M. (1991). La productividad de la Administración Española: Un Análisis Comparativo. Madrid: Ministerio de Economía y Hacienda.

CASANOVA, M.A. (1987). «La evaluación educativa, a debate». Comunidad Educativa, $155,(23-27)$.

CELORRIO, R. (1984). Evaluación del rendimiento en la enseñanza superior a distancia: El Centro Asociado de la UNED de Soria. Tesis Doctoral. Ciencias de la Educación. Madrid: UNED.

COOK, T.D.; ReICHARDT, C.S. (1986). Métodos cualitativos y cuantitativos en investigación evaluativa. Madrid: Morata.

CROMBACH, L.J. (1981). Toward reform of programm evaluations. San Francisco: JosseyBass.

DE MIGUEL, M. (1991). La evaluación de las instituciones universitarias. Madrid: Consejo de Universidades.

FERNÁNDEZ SIERRA, J. (1992). Evaluación cualitativa de programas de educación para la salud. Málaga: Ediciones Aljibe. 
GUBA, E.G. (1985). Toward a Metodology of Naturalistic Inquiry in Educational Evaluation. Los Angeles: Center for the Study of Evaluation, UCLA.

House, E.R. (1979). "Coherence and credibility: The esthetics of evaluation". Educational evaluation and policy analysis, 1, 5 .

MCDONALD, B. (1985). Democracy and Evaluation. University of East Anglia.

MCDONALD, F; ELIAS, P. (1976). Executive summary report: Beginning teacher evaluation study, Phase II. Princeton, NJ.: Educational Testing Service.

PARLETT, M.R.; HAMILTON, D. (1987). "Evaluation in illumination: A new approach to the study of innovative programmes". En HAMILTON, D. y otros (eds.). O.c.

PÉrez Gómez, A. (1985). "Modelos comtemporáneos de evaluación». En GiMENo, J.; PEREZ, G. (comp.). La enseñanza, su teoría y su práctica. Madrid: Akal/Universidad.

Roses (1984). Citado en BELTRAN, M. (1991). La productividad de la Administración Española: Un Análisis Comparativo. Madrid: Ministerio de Economía y Hacienda.

SABIRON, F. (1990). Evaluación de Centros Docentes. Modelo, aplicaciones y guia. Zaragoza: Central de Ediciones, S.L. Col. Documentos de trabajo en educación.

SANTOS, M.A. (1990). Hacer visible lo cotidiano. Teoría y práctica de la evaluación cualitativa de centros escolares. Madrid: Akal.

SCRIVEN, M.S. (1975). "Evaluation bias and its control». Occasional Paper Series, núm. 4. Universidad de Western Michigan. Centro de Evaluación.

STAKE, R.E. (1975). "Programm evaluation, particularly responsive evaluation". Occasional Paper Series, núm. 5. University of Western Michigan. Evaluation Center.

STAKE, R.E. (1981). "Setting standars for educational evaluators». Evaluation News, 2, 2, (148-152).

STUFFlebeAM, D.L.; SHINKFIELD, A.J. (1987). Evaluación sistemática: Guía teórica y práctica. Madrid: Paidós-MEC. 